\title{
Granular B-acute lymphoblastic leukemia: the characterization of its morphology, immunophenotype and ultrastructure

Huanling Wư ${ }^{1}$, Bing Liu², Boqin $\mathrm{Li}^{3}$, Yong Wang${ }^{1}$, Haibo Wang ${ }^{1}$, L. Jeffrey Medeiros ${ }^{4}$, Bingchang Zhang ${ }^{1, \#, ~ W e i ~ W a n g ~}$ Department of Clinical Laboratory Medicine, Shandong Provincial Hospital Affiliated Shandong University, Jinan, Shandong, China, 250021;

2 Department of Clinical Laboratory, The Tumor Hospital of Zhengzhou University, Zhengzhou, Henan, China, 450008 3 Shandong WEI-YA Biotech Company, Jinan, Shandong, China, 250012

${ }^{4}$ Department of Hematopathology, The University of Texas MD Anderson Cancer Center, Houston, U.S.A, 77030

$$
\text { Background }
$$

The presence of cytoplasmic granules in the blasts is a well-known feature of acute leukemia with myeloid differentiation, whereas B-acute lymphoblastic leukemia (B-ALL) with cytoplasmic granules in blasts is rare and may potentially be misdiagnosed as acute myeloid leukemia. Although some literatures proposed a criterion for granular ALL with more than $5 \%$ of lymphoblasts having three or more clearly defined azurophilic granules greater than 0.5 $\mu \mathrm{m}$, the nature and component of granules in Blymphoblasts are not fully understood. In this study, we describe a rare case of hypergranular B-cell acute lymphoblastic leukemia (ALL) and characterized the nature of granules using electronic microscopy.

$$
\text { Objective }
$$

Described a case of hypergranular B-ALL and characterized granules using electronic microscopy.

$$
\text { Result }
$$

1. A 41-year-old woman presented with a WBC count of $2.63 \times 10^{9} / \mathrm{L}$, hemoglobin of $106 \mathrm{~g} / \mathrm{L}$ and platelet of $162 \times 10 \% / L$

2. The marrow aspirate smears showed $68 \%$ blasts and the majority of blasts had abundant large and purple cytoplasmic granules (Fig 1A).

3. The granules in the blasts were negative for myeloperoxidase (MPO) (Fig1B), and positive for $\alpha$-naphthol acetate esterase ( $\alpha$ NAE) (Fig1C) and Periodic acid-Schiff reaction (PAS) (Fig1D).

4. By flow cytometric analysis, the blasts were positive for B-cell (Fig 1E).
5. Under electron microscope, these cytoplasmic granules presented as "azurphilic" granules by Wright-Gimesa stain, were oval-shaped, membrane-enclosed and low-electrondensity vesicular structures which were most consistent with swollen endoplasmic reticulum (Fig 2A-D). Electric microscopy analysis of a case of classic agranular B-ALL was also performed, and these low-density vesicular structures are

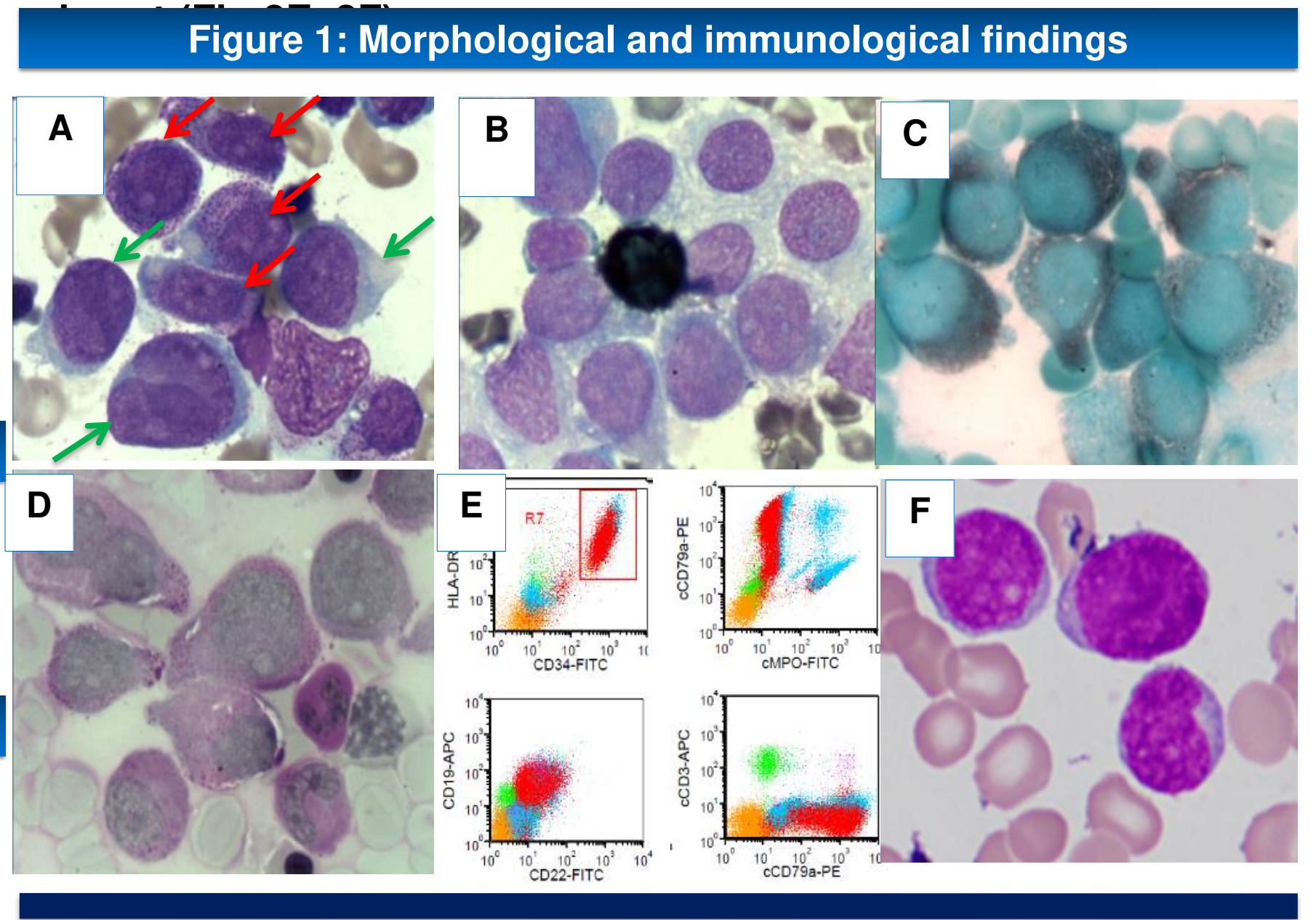

Fig A, Bone marrow smear shows two different types of lymphoblasts, one with abundant large cytoplasmic granules (red arrow, predominant population) and the other with no granules (green arrow, minor population). (Wright-Giemsa stain, $\times 1000$ ). Granular lymphoblasts in bone marrow smear are completely negative for MPO (B, myeloperoxidase stain, $\times 1000$ ), strong positivity to $\alpha-N A E$ staining (C, $\alpha-N A E$ stain, $\times 1000)$ and strong PAS positivity (D, Periodic acid-Schiff stain, $\times 1000)$. E, flow cytometry shows the granular lymphoblasts are positive for CD34, CCD79a, CD19,CD22, and negative for CMPO and CCD3. F, a classic B-ALL shows no cytoplasmic granules (Wright-Giemsa stain, $\times 1000$ ).

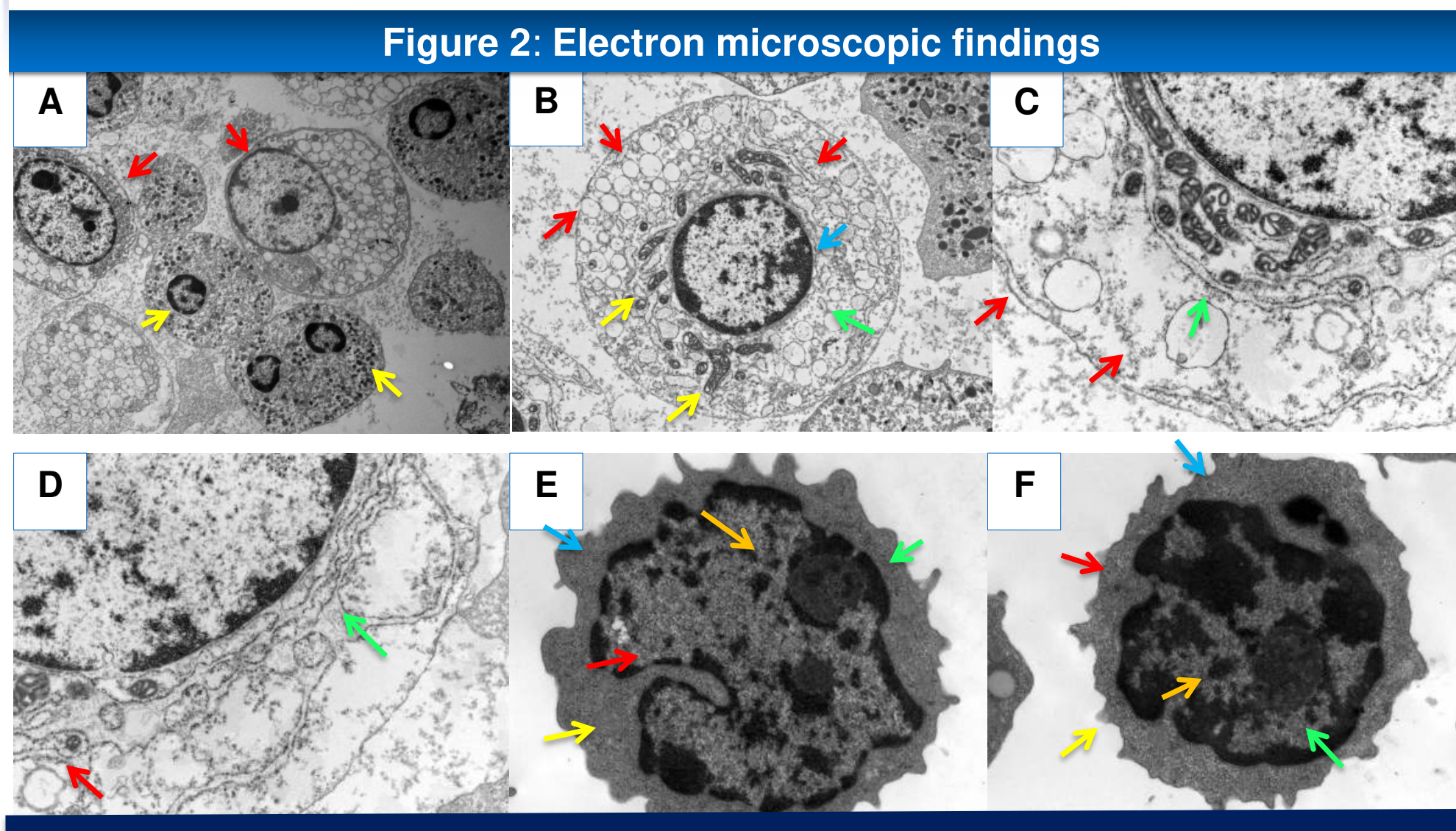

Fig A, Abundant membrane-enclosed vesicular substructures in granula lymphoblasts (red arrows) are present in the cytoplasm. Myeloid cells (yellow arrows) are captured also (original magnification $\times 5,000$ ). B, granular lymphoblast presents with abundant membrane-enclosed vesicular structures (red arrows). Mitochondria (yellow arrows) are presented adjacent to the nuclei. Rough endoplasmic reticulum (green arrow) and ribosome (blue arrow) are also identified (original magnification $\times 12,000)$. C, vesicular structures corresponding to cytoplasmic granules (red arrows), mitochondria (yellow arrow), flat rough endoplasmic reticulum (green arrow) and ribosomal (blue arrow) in the cytoplasm(original magnification $\times 30,000$ ). D, granular lymphoblast shows swollen fusion of endoplasmic reticulum (green arrow), and a few membrane-enclosed vesicular organelles (red arrow) (original magnification $\times 30,000$ ). $E$ and $F$, in comparison, a classic $B-A L L$ with no cytoplasmic granules is shown here. The lymphoblast presents with round body, pseudopodium(green arrow), irregular nuclei with deep grooves (red arrow), prominent nucleolus (orange arrows), mitochondria ( yellow arrow), ribosome (blue arrow) in the cytoplasm No cytoplasmic ( yellow arrow), ribosome (blue arrow) in the

Granular B-ALL is rare but the presence of cytoplasmic granules can cause diagnostic challenges. The nature of cytoplasmic granules is controversial. In our study, our electronic microscopic analysis suggests they are most consistent with endoplasmic reticulum

\section{References}

1. Cerezo L, Shuster JJ, Pullen DJ et al. Laboratory correlates and prognostic significance of granular acute lymphoblastic leukemia in children. A pediatric oncology group study. Am J Clin Pathol, 1991;526-53

2. Song JY, Khojeini EV, Dwyre DM, Jonas BA. B lymphoblastic leukemia with granules mimicking acute myeloid leukemia. Int $J$ Hematol 2015;102;251-252.

3. Fradera J, Velez-Garcia E, White JG. Acute lymphoblastic leukemia with unusua cytoplasmic granulation: A morphologic, cytochemical, and ultrastructural study. Blood 1986;68;406-411. 